\title{
Cosmic Ray Research in Armenia
}

\author{
A. A. Chilingarian ${ }^{a}$, R. G. Mirzoyan ${ }^{b}$, and M. Z. Zazyan ${ }^{a}$ \\ ${ }^{a}$ Alikhanian Yerevan Physics Institute, Yerevan, Armenia \\ ${ }^{b}$ Max-Planck-Institute for Physics, Munich, Germany \\ Received March 9, 2009
}

\begin{abstract}
Cosmic Ray research on Mt. Aragats began in 1934 with the measurements of East-West anisotropy by the group from Leningrad Physical-Technical Institute and Norair Kocharian from Yerevan State University. Stimulated by the results of their experiments, in 1942 Artem and Abraham Alikhanian brothers organized a scientific expedition to Aragats. Since that time physicists were studying Cosmic Ray fluxes on Mt. Aragats with various particle detectors: mass spectrometers, calorimeters, transition radiation detectors, and huge particle detector arrays registering protons and nuclei accelerated in most violent explosions in Galaxy. Latest activities at Mt. Aragats include Space Weather research with networks of particle detectors located in Armenia and abroad, and detectors of Space Education center in Yerevan.
\end{abstract}

PACS numbers: 96.50.S-

DOI: $10.3103 / \mathrm{S} 106833720905003 \mathrm{X}$

Key words: cosmic rays, particle detectors, cosmic weather, history

\section{INTRODUCTION}

Information on the particles of highest energies bombarded the Earth's atmosphere significantly provides vast information on most violent processes in the Universe. One of the "main players" reflecting physical processes in stellar systems are particles and stripped nuclei reaching the Earth from interstellar space and from Sun: so-called Galactic and Solar Cosmic Rays (GCR and SCR). These "primary" Cosmic Rays (CR) were discovered almost 100 years ago by the ionization effects of the secondary fluxes (particle showers) produced in their interactions with the terrestrial atmosphere. Exploiting different physical processes of shower interaction with the atmosphere (particle multiplication, fluorescence, Cherenkov light emission in atmosphere and in water, acoustic waves, and radio waves emissions) different experimental techniques were developed to detect cosmic rays above and on the Earth's surface, underground and underwater. Fifty years ago with launch of the first satellite on 4 October 1957 experiments in space directly detected primary cosmic rays and confirmed that our nearest star, the Sun, is a particle accelerator.

Direct measurements of particle fluxes by facilities on board satellites and balloons provide excellent charge and energy resolution but, due to severe limitation of payload and the weak flux of high-energy $\mathrm{CR}$, perform measurements in $\mathrm{keV}$ to $10 \mathrm{GeV}$ energy regions. In hundreds of $\mathrm{TeV}-\mathrm{PeV}$ region now and in the nearest future only surface-based techniques of detecting secondary particle showers can provide data on energy and types of primary particles.

One of the first permanent high-mountain research stations was established on Mt. Aragats 65 years ago. The Aragats and Nor-Amberd research stations of the Cosmic Ray Division (CRD) of the Yerevan Physics Institute (YerPhI) named after A. Alikhanian are located on slopes of Mt. Aragats, the highest peak of modern Armenia (see Figs. 1 and 2), at 3200 m and 2000 m elevation, respectively. The scientific history of cosmic ray research at Aragats can be traced back to 1934 when a group from Leningrad Physical-Technical Institute and Norair Kocharian from Yerevan State University (YSU), measured the East-West cosmic ray anisotropy [1]. These measurements stimulated the interest of famous physicists, the brothers Artem and Abraham Alikhanian (see Fig. 3), who organized a scientific expedition to Aragats in 1942. Since then, expeditions on Aragats continued uninterruptedly, in spite of World War II, insufficient funding, and electricity and fuel shortages during the recent history of Armenia.

In the 40's and 50's cosmic rays were the main source for information about the properties of elementary particles. Later CR investigations have led to new, modern branches of physics named "Astroparticle Physics", "High-Energy Astrophysics" and "Space Weather". The most important data 


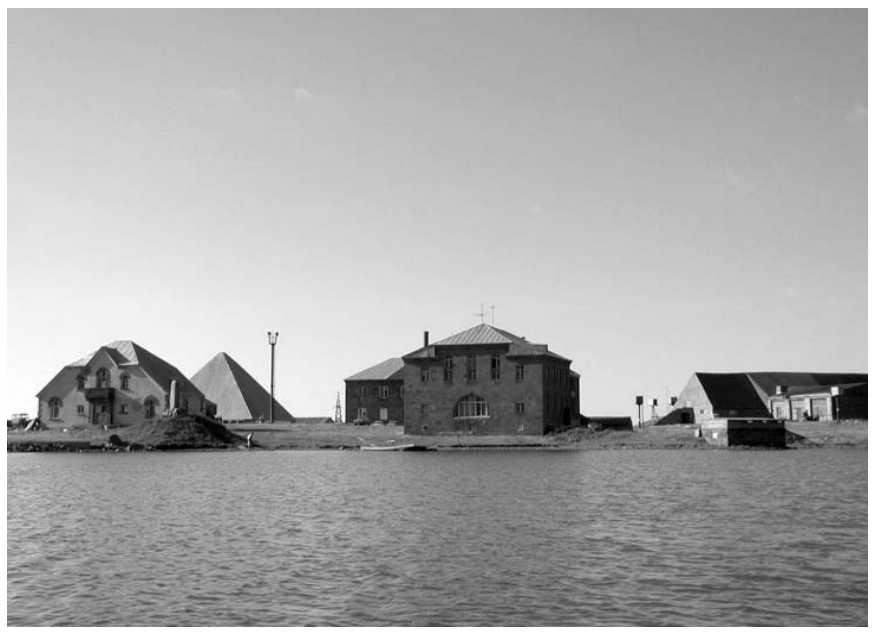

Fig. 1. Aragats research station (altitude $3200 \mathrm{~m}$ ).

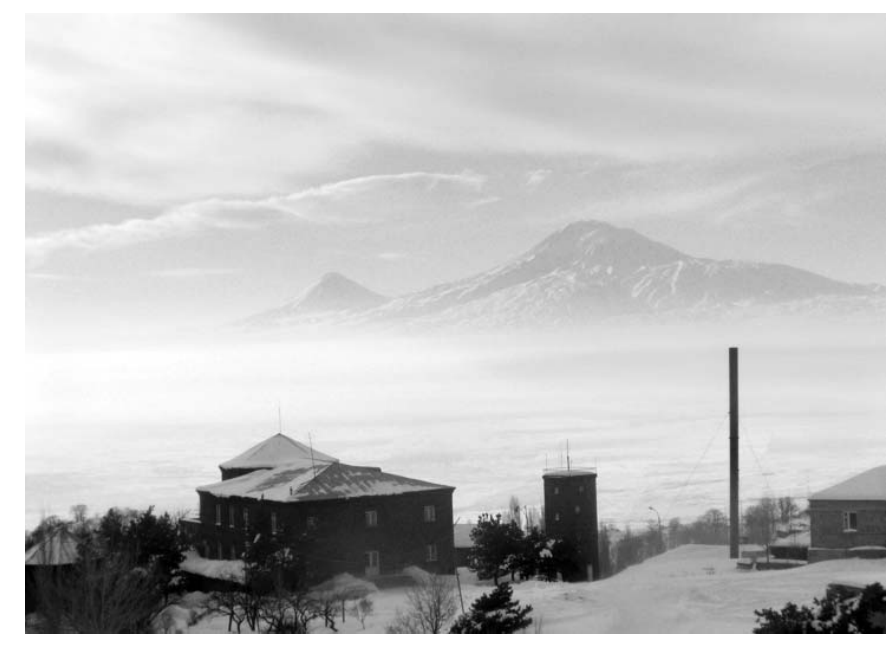

Fig. 2. Nor-Amberd research station (altitude $2000 \mathrm{~m}$ ).

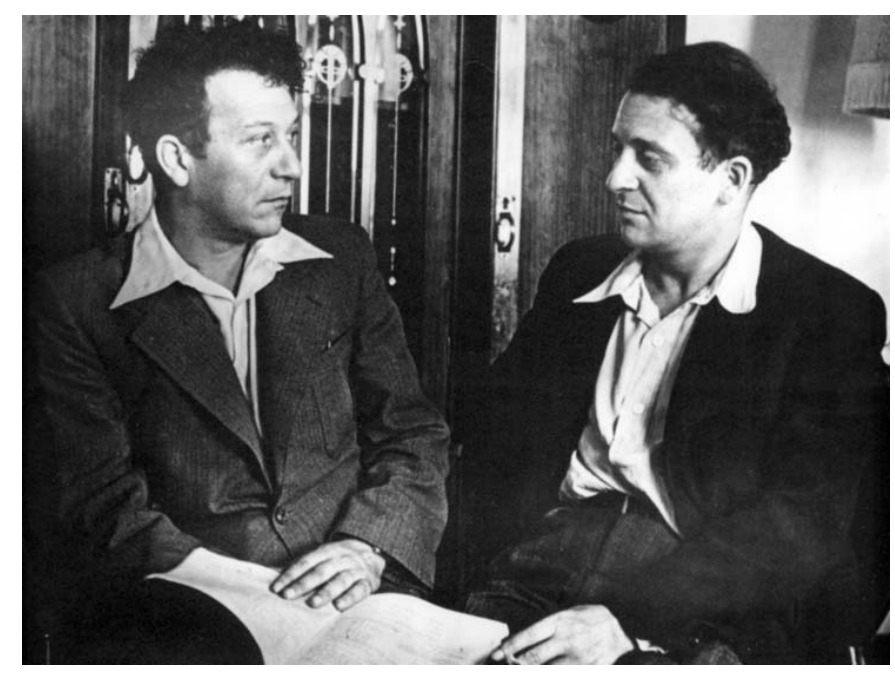

Fig. 3. Abraham Alikhanov (left) and Artem Alikhanian. 
and achievements of Cosmic Ray research at Aragats can be itemized as follows:

- 1942 - First expedition to Aragats;

- 1943 - Establishment of the Physical-Mathematical Institute of the Armenian Academy of Sciences; now Yerevan Physics Institute after Artem Alikanian;

- 1945-1955 - Foundation of Aragats high-mountain research station. Experiments at Aragats with mass-spectrometer of Alikhanian-Alikhanov: investigations of the composition of secondary CR (energies <100 GeV); exploration of the "third" component in CR; observation of particles with masses between $\mu$-meson and proton;

- 1957 - Installation of the ionization calorimeter, detection of particles with energies up to $50 \mathrm{TeV}$;

- 1960 - Foundation of the Nor-Amberd high-mountain research station;

- 1970 - Modernization of the wide-gap spark chambers;

- 1975 - Experiment MUON: measurements of the energy spectrum and charge ratio of the horizontal muon flux;

- 1975 - Installation of the neutron supermonitors at the Aragats and Nor-Amberd research stations;

- 1977 - Experiment PION: measurements of pion and proton energy spectra and hadron interaction phenomenological parameters;

- 1981-1989 - ANI Experiment: commence of MAKET-ANI and GAMMA surface detector arrays for measuring cosmic ray spectra in the "knee" region $\left(10^{14}-10^{16} \mathrm{eV}\right)$;

-1989-1992 - Design and tests of the system of atmospheric Cherenkov telescopes, introduction of multivariate methods for signal detection from $\gamma$-ray point sources;

-1993-1996 - Development of new methodology of multivariate, correlation analysis of data from Extensive Air Shower detectors, event-by-event analysis of shower data from KASCADE experiment; classification of primary nuclei;

- 1996-1997 - Renewal of cosmic ray variation studies at Aragats: installation of the Solar Neutron Telescope and resumption of the Nor-Amberd Neutron Monitor;

- 2000 - Foundation of the Aragats Space Environmental Center (ASEC) - for Solar Physics and Space Weather research; measurements of the various secondary fluxes of cosmic rays; inclusion of the large surface arrays in monitoring of the changing fluxes of secondary cosmic rays;

- 2003 - Detection of the intensive solar modulation effects in September-November in the low-energy charged particle, neutron and high-energy muon fluxes;

- 2004 - Measurement of the spectra of heavy and light components of GCR, observation of very sharp "knee" in light nuclei spectra and absence of "knee" in heavy nuclei spectra;

- 2005 - Measurements of highest energy protons in Solar Cosmic Rays (GLE 70 at 20 January; detection of Solar protons with $E>20 \mathrm{GeV}$ );

- 2007 - Starting of SEVAN (Space Environmental Viewing and Analysis Network) - a new type of world-wide network of particle detectors for monitoring of geophysical parameters.

\section{THE MASS-SPECTROMETRIC PERIOD \\ OF SCIENTIFIC RESEARCH ON MT. ARAGATS}

The history of scientific research on Mt. Aragats can be divided into several periods. The first - massspectrometric period - lasted about 15 years. Experiments with magnetic spectrometer designed by the Alikhanian brothers lead to the discovery of protons in CR [2] and narrow air showers [3]. According to the viewpoint of that time, CRs were believed to have a pure electromagnetic origin [4], therefore the presence of protons in CR strongly contradicted the established concepts. The origin of narrow showers could not be electromagnetic because of their great penetrability. Later narrow showers were thoroughly studied with the Aragats Ionization Calorimeter [5].

Using the Alikhanian-Alikhanov magnetic spectrometer N. Kocharian obtained the energy spectra of muons and protons with energies up to several $\mathrm{GeV}$ [6]. Till now this data remain one of the best measurements of the secondary cosmic ray fluxes at mountain altitudes.

The mass spectrometer method, performing the simultaneous measurement of the momentum and absorption length of charged particles, provided the effective particle mass analysis. This method provided the first evidence of the existence of particles with masses ranging from $\mu$-meson to proton. However, only some of the many peaks in mass distributions measured at Aragats were later verified to be "real" particles and became known as $\pi$ - and K-mesons. Other "particles" with masses heavier than 
$\mu$-meson, including so-called varitrons [7], "discovered" using the Aragats mass-spectrometer, turned to be artifacts due to fluctuations in the mass distributions. Nonetheless, the discussion on varitrons led to several excellent experimental and theoretical investigations and Alikhanian brothers' idea about a variety of elementary particles became very popular among physicists all over the world, making the Aragats research station one of the most important centers of cosmic ray physics. It should be mentioned that defining the reliability of peaks in one- and two-dimensional distributions is still one of the most important and complicated problems in High-Energy Physics and Astrophysics. Also nowadays there are many groups, using sophisticated mathematical methods, which cannot avoid mistakes and reported discoveries based on the fake peaks (see, for example, discussion about "discovery" of pentaquark [8]).

\section{CALORIMERTRIC MEASUREMENTS ON Mt. ARAGATS}

The second phase of scientific research on Mt. Aragats, calorimetric measurements, covers the period from 1958 to 1970. The mass spectrometric method had reached its energy limit by that time. In 1958 a group of scientists from the Institute of Nuclear Physics of Moscow State University and Yerevan Physics Institute (team leader Naum Grigorov) installed the first ionization calorimeter at the Aragats station. Experiments with ionization calorimeter at Aragats proved the energy-dependence of the effective inelastic cross-section of the hadron interaction with nuclei. This fact was later confirmed by direct measurements on Proton satellites [9] and accelerator experiments. The ionization calorimeter also detected another interesting result concerning the peculiarities of multiparticle production of high-energy pions [10], which was later (1990) registered as a discovery in USSR: in some cases only few $\pi^{0}$-mesons, generated in the interaction with atmospheric nuclei, "take away" almost the entire energy of the primary particle. The authors of this discovery were Kh. Babayan (deputy-director of YerPhI in 1956-1969), Naum Grigorov, Erik Mamijanyan (head of the Cosmic Ray Division of YerPhI in 1969-1992) and Vladimir Shestoperov.

The Nor-Amberd station, which started operation in 1960 (see Fig. 2) at the altitude of $2000 \mathrm{~m}$, considerably enlarged the possibilities for studying high-energy cosmic ray hadrons and their interaction with different nuclei (head of laboratory in 1960-1986- Gerasim Marikyan).

At that time physicists from various scientific institutions of the Soviet Union participated in the investigations at the Armenian mountaines, also scientists from USA, France, Japan and Great Britain visited high-altitude stations. The method of wide-gap spark chambers was intensively investigated in YerPhI in late 50-s. The prestigious Lenin Prize was awarded to Artem Alikhanian and Tina Asatiani (head of the muon laboratory of YerPhI in 1960-1987) in collaboration with groups of Russian and Georgian physicists for developing the wide-gap spark chamber techniques.

In 1968-69 a system of proportional counters was added to the Aragats ionization calorimeter. Using this facility, the neutron component of cosmic rays at mountain altitude was measured by E. Mamijanyan and his colleagues [11].

$\mathrm{Kh}$. Babayan in early 70 -s started his research of CR variations by installing neutron supermonitors at the Aragats and Nor-Amberd research stations, which served as a basis for creating a unique center of cosmic ray monitoring in the "new history" of Aragats.

\section{HIGH-ENERGY ASTROPHYSICS}

During the next period (1970-1980) experiments PION [12] and MUON [13] measured fluxes of secondary cosmic rays and some phenomenological characteristics of strong interactions. The team leaders of the experimental groups were Vahram Avakyan (head of the Aragats station from 1963 till 1993) and Tina Asatiani, respectively. PION was a unique facility [14], which includes transition radiation detection system for particle identification, created by Albert Oganesian's group (head of laboratory from 1978 till 1996) and an ionization calorimeter for particle energy estimation.

The muon magnetic spectrometer for studying near-horizontal high-energy muons was equipped with coordinate measuring systems based on the wire spark chambers and wide-gap spark chambers, thus increasing the range of reliable muon momentum measurement up to $\sim 2.5 \mathrm{TeV}$. Both experiments used modern numerical algorithms and on-line computers for data analysis. One of the first soviet computers M220 was used to calculate the horizontal muon energy spectrum. The PION experiment used the first Armenian minicomputer NAIRI-2 for data acquisition.

In the 80 's it became clear that larger detectors are necessary for the research of primary cosmic ray fluxes. The ANI experiment on Mt. Aragats [15] met all these requirements. It was intended to register electrons and muons of Extensive Air Showers (EAS) by a system of surface scintillators; interactions of 
hadrons from EAS core with the world's largest calorimeter (surface area $1600 \mathrm{~m}^{2}$ ); high-energy muons by a huge underground muon detector and huge magnetic spectrometer (surface area $40 \mathrm{~m}^{2}$ ). The ANI experiment was designed in cooperation with the Lebedev Physics Institute of USSR Academy of Sciences under the guidance of USSR Ministry of Medium Machinery (presently, Federal Nuclear Energy Agency of the Russian Federation). The experiment leaders were Sergey Nikolsky (director of the Division of Nuclear Physics and Astrophysics of the Lebedev Physics Institute) and Erik Mamijanyan.

The ANI complex was not completed because of the collapse of the USSR, followed by the collapse of the Armenian economy, but 2 surface particle arrays MAKET-ANI (experiment leader Gagik Hovsepyan, see details in [16]) and GAMMA (experiment leader Romen Martirosov, see details in [17]) made significant contribution to the "knee" region physics. For selecting the proper model of the CR origin one has to measure the partial energy spectra of the different groups of primary nuclei, i.e. perform the classification of the primary nuclei by highly smeared EAS information content. These very complicated tasks become feasible after developing the nonparametric multivariate methodology of data analysis by Ashot Chilingarian in 1989 (head of CRD since 1993).

Event-by-event-analysis of EAS data, using Bayesian and Artificial Neural Network (ANN) information technologies $[18,19]$ helped to obtain the energy spectra of light and heavy primary nuclei from MAKET-ANI experiment and also 3 partial spectra, corresponding to light, intermediate and heavy nuclei groups from KASCADE experiment [20]. MAKET-ANI data [21, 22] demonstrates the existence of a sharp knee in the light component, and no evidence of knee in the heavy component up to $\sim 3 \times 10^{16} \mathrm{eV}$ (see Fig. 4). The available world data confirm these results. In the KASCADE experiment, the position of the knee shifts towards higher energies with increasing mass number [23]. In HEGRA experiment [24] a steepening of the light mass group spectrum was detected. In EAS-TOP [25] the light nuclei group also demonstrates a sharp knee. Therefore, EAS evidence on the galactic CR origin consists in establishing charge dependent acceleration of CR in general agreement with model of shock acceleration in the blast waves of supernova explosions. Further observations made by orbiting in space gamma-ray observatories and ground-based Atmospheric Cherenkov Telescopes (ACTs) also point on the Supernovae Remnant (SNR) as one of the major cosmic ray sources.

After publishing the final papers the MAKET-ANI detector ceased operation in 2007. The scintillators are used now for monitoring changing fluxes of low-energy charged CRs. Arrangement was made also for making a test facility for the new precise timing system for a new large EAS array for measuring CRs far beyond the knee, now under consideration at CRD.

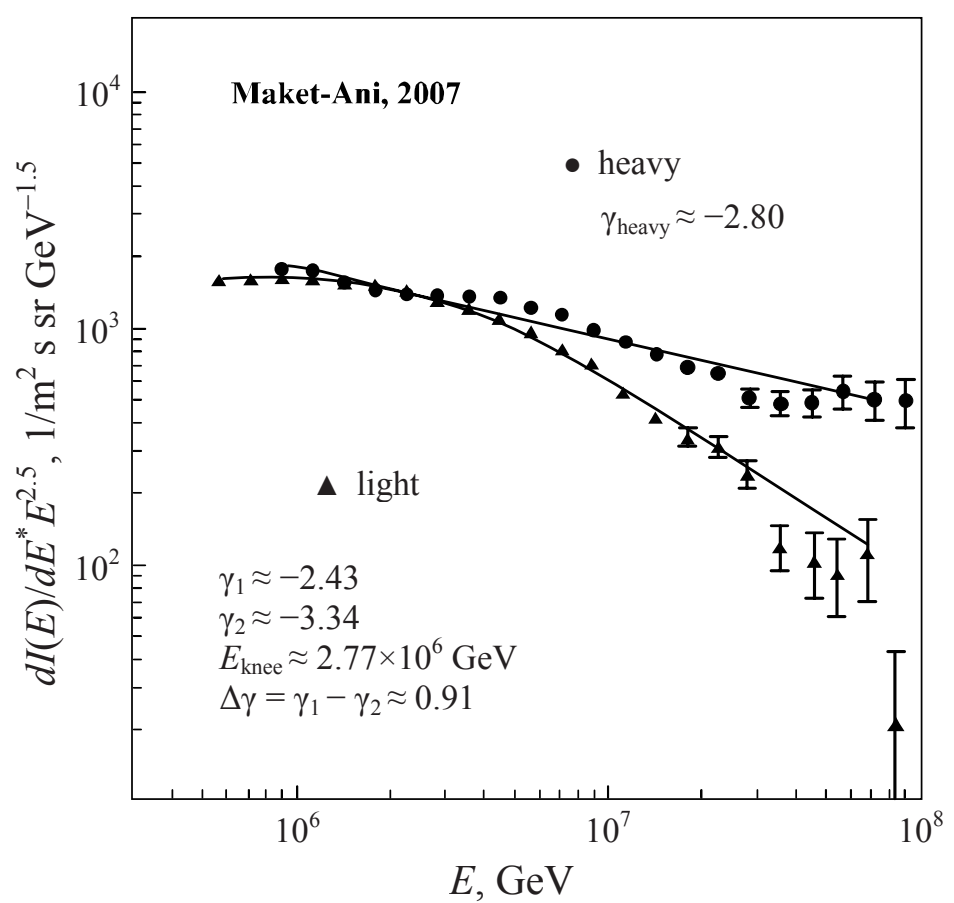

Fig. 4. Differential spectra of light and heavy nuclei groups of primary flux as measured by the MAKET-ANI surface array. 
Direct evidence of shock acceleration in SNR shells can be deduced from joint detection of young SNRs in X-and $\gamma$-rays. To prove that the young supernovae remnant RX J1713.7-3946 is a very efficient proton accelerator Uchiyama with colleagues [26] include in the analysis information on broadband X-ray spectra (from 0.4 to $40 \mathrm{keV}$ ) measured by the Suzaku satellite [27] and on high-energy $\gamma$-ray spectra (extending over $10 \mathrm{TeV}$ ) measured by HESS Atmospheric Cherenkov Telescope [28]. They exclude the inverse Compton origin of detected high-energy $\gamma$-quanta, and taking into account the $\mathrm{TeV}-\mathrm{keV}$ correlations validate the hadronic model of detected $\gamma$-rays. Thus, the joint analysis of X-ray maps from Chandra and X-ray spectra from Suzaku satellites with high-energy $\gamma$-ray spectra measured by HESS ACT provide very strong arguments for the acceleration of protons and nuclei of $1 \mathrm{PeV}$ and beyond in young SNR shells.

Armenian physicists have a significant impact in the development of the ACT technique. Pioneering system of ACTs on Canarias (HEGRA) followed by large ACTs HESS in Namibia and MAGIC at Canarias designed and operated by international collaborations with the participation of Armenian physicists.

In 1985 design and construction of the first system of ACTs for the ANI experiment at Aragats started at YerPhI. The telescopes comprised tessellated reflectors of $3 \mathrm{~m}$ diameter and an imaging camera in the focal plane of 37 pixels based on FEU-130 type Soviet PMTs of bialkali type. High-quality glass mirrors with quartz protection, equatorial mounts of the telescopes, the imaging cameras and DAQ electronics also were prepared at YerPhI workshops. The gamma-ray group was led by Felix Aharonian and Razmik Mirzoyan. The group started measuring cosmic ray signals at the Nor-Amberd research station and calibrating the telescope for the first measurements of the Crab Nebula when the collapse of the former Soviet Union stops experimental activities. Fortunately, the Armenian scientists with German physicist O. Alkoffer prepare a proposal to install the same system of ACTs on a newly created HEGRA (HighEnergy Gamma-Ray Astronomy) cosmic ray detector on the Canary Islands (Las Palmas). Already prepared devices and materials for the construction of the 5 telescopes have been shifted from Armenia via Germany to Las Palmas and the construction started in 1991. In 1992 the first HEGRA telescope measured gamma-rays from Crab Nebula [29].

That was the first significant confirmation of the discovery of the $10 \mathrm{~m}$ diameter Whipple telescope in Arizona, USA. In 1993 second telescope was build and operated in stereo mode with the first one and later on 4 more telescopes were added to the system. The HEGRA telescopes were operated until 2002 and provided a rich harvest of gamma-sources. The contribution of Armenian physicists in HEGRA was very significant because of their leading role both in the techniques of IACTs as well as their theoretical work on the very frontier of gamma-astronomy.

After termination of HEGRA the astrophysicists from the collaboration continued to build new advanced instruments. Already in 1994 the $17 \mathrm{~m}$ diameter MAGIC telescope, intending to investigate gamma-rays below $300 \mathrm{GeV}$ down to energies of $30 \mathrm{GeV}$ was proposed by Razmick Mirzoyan. An international collaboration was formed and in 1998 it became an official project in Max-Planck-Institute for Physics (MPI) in Munich. YerPhI and several institutions in Germany, Spain, Italy, Switzerland and Finnland became members of the MAGIC collaboration. The first MAGIC telescope was built in Las Palmas in 2001-2003 and has been in operation since 2004. The second MAGIC telescope was built at $85 \mathrm{~m}$ distance from the first one and will operate together with the first one in fall, 2009.

The other part of HEGRA collaboration continues its research with $10 \mathrm{~m}$ diameter class telescopes, with advanced optics and electronics. A new array, initiated in 1997 by Felix Aharonian, under the name HESS, is comprised of 4 telescopes of $12 \mathrm{~m}$ diameter and was built by an international collaboration, mostly from Germany and France, in Namibia in 2001-2003. Scientists from YerPhI also became member in HESS. HESS collaboration intends to complete their array with one $28 \mathrm{~m}$ diameter very large telescope in 2009.

The number of sources increased from $\sim 20$ to more than 80 just in 3-4 years and very interesting publications, more than 70 by now, appeared in peer-refereed journals, also in such famous ones as Science and Nature. It is expected that both telescopes together will increase the number of sources to $\sim 100$ just in the next 2-3 years and finally long-standing questions of cosmic rays, astrophysics, and astroparticle physics can be understood and answered.

\section{SOLAR PHYSICS AND SPACE WEATHER RESEARCH}

Cosmic Rays are accelerated not only in the depths of galaxies but also by our nearest star, the Sun. Strong solar flares sometimes accelerate particles in the $\mathrm{MeV}-\mathrm{GeV}$ range to intensities more than the total galactic flux reaching terrestrial atmosphere. Solar particles interact with the magnetosphere, ionosphere 
and the atmosphere, thus influencing the near-Earth environment and abruptly changing the "space weather", seriously impacting space-born and Earth-based technologies. Space storms can harm astronauts in space and cause excessive radiation exposure for aircraft crew. Space weather changes very fast, the intensity of X-ray radiation and particles of high energies can greatly increase in a few seconds. Protons and nuclei, which penetrate microscopic electronic devices create additional currents and change the state of the electronic circuits, generating false commands and damaging on-board management systems. Electron fluxes, rushing through the atmosphere, create polar flares and induce currents on the surface of conductors, which cause pipeline corrosion and damage transformers at electric stations. Our civilization heavily depends on space-based technologies, including telecommunication, navigation, disaster warning, weather forecasting, military systems, etc. For this reason, space weather research attracts more and more scientists. At the end of last century USA, Canada, Europe and Japan adopted national programs to study space weather and to create reliable forewarning services. CRD physicists are contributed to this important endeavor.

Starting in 1996 we have been developing various detectors to measure fluxes of different components of secondary cosmic rays. In 1996 we restarted our first detector - the Nor-Amberd Neutron Monitor 18NM64. A similar detector started to take data at the Aragats research station in autumn 2000. A Solar Neutron Telescope (SNT) has been in operation at the Aragats research station since 1997 [30], as a part of the worldwide network coordinated by the Solar-Terrestrial laboratory of Nagoya University. In addition to the primary goal of detecting the direct neutron flux from the Sun, the SNT also has the ability to detect charged fluxes (mostly muons and electrons) and roughly measure the direction of the incident muons. Another monitoring system is based on the scintillation detectors of the Extensive Air Shower surface arrays, MAKET-ANI and GAMMA, located on Mt. Aragats. The charged component monitoring system at the Nor-Amberd research station started operation in 2002. Our Data Acquisition (DAQ) system was modernized in 2005. Modern electronics was designed to support the combined neutron-muon detector systems as well as measurement of the environmental parameters (temperature, pressure, humidity). Microcontroller-based DAQ systems and high-precision time synchronization of the remote installations via Global Positioning System (GPS) receivers are crucial ingredients of the new facilities on Mt. Aragats. Information on changing secondary particle fluxes, measured by hundreds of detecting channels, is used for the enumerating solar modulation effects during large solar explosions.

20.01.2005 (3 min)

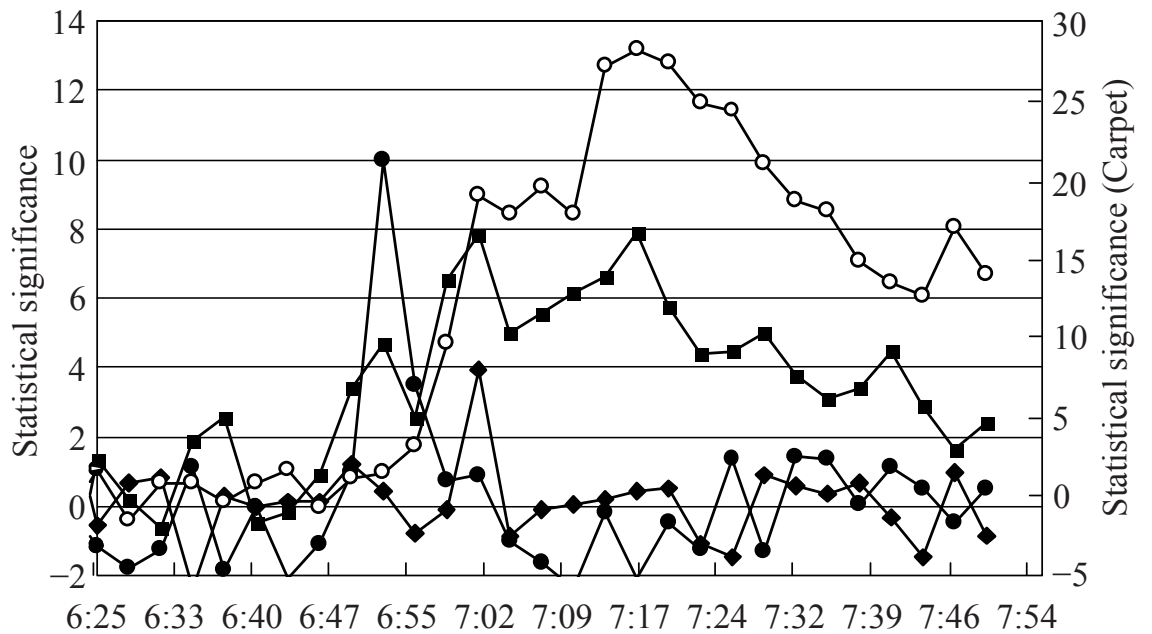

UT

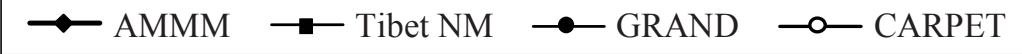

Fig. 5. Time series of 3 minute count rates of secondary muons and neutrons detected at 20 January 2005. Note the second peak at 7:02 detected by CARPET (most probable energy of primary protons $\sim 10 \mathrm{GeV}$ ), Tibet NM (most probable energy of primary protons $\sim 13 \mathrm{GeV}$ ) and AMMM (most probable energy $>20 \mathrm{GeV}$ ).

The Aragats Space Environmental Center (ASEC, [31, 32]) operating since 2000, provided one of the most detailed coverages of the violent events of the $23^{\text {rd }}$ solar activity ending in 2008. One of the most exciting results obtained recently at Mt. Aragats is the discovery of protons of highest energies (greater 
than $20 \mathrm{GeV}$ ) accelerated on the Sun during space-era largest Ground Level Enhancement (GLE) $[33,34]$.

On 20 January 2005, during the recovery phase of the Forbush decrease, a long lasting X-ray burst occurred near the west limb of the Sun (helio-coordinates: 14N, $67 \mathrm{~W}$ ). The start of the X7.1 solar flare was at 06:36 and maximum of the X-ray flux at 7:01. The fastest (relative to X-ray start time) GLE event of $23^{\text {rd }}$ cycle was detected by space-born and surface particle detectors a few minutes after the flare onset. The start of GLE was placed at 6:48; the maximal amplitude of 5000\% recorded by NM at the South Pole is the largest increase ever recorded by neutron monitors. ASEC monitors detected significant excess of count rates at 7:00-8:00 UT. From 7:02 to 7:04 UT, the Aragats Multichannel Muon Monitor (AMMM) detected a peak with the significance of $\sim 4 \sigma$. This was the first time that we detected a significant enhancement of the $>5 \mathrm{GeV}$ muons coinciding with the GLE detected by the world-wide networks of Neutron Monitors. Detailed statistical analysis of the peak [35] proves a non-random nature of the detected enhancement. This short enhancement (see Fig. 5) exactly coincides in time with peaks from Tibet Neutron Monitor [36], Tibet Solar Neutron Telescope [37] and the Baksan scintillator surface array [38] (Fig. 5). Another surface array (GRAND, located in the Western hemisphere) demonstrated a very large peak $\sim 10$ minutes earlier [39].

The differential energy spectra of the SCR protons at 7:02-7:04 UT measured by the space born spectrometers and surface particle detectors covers more than 3 orders of magnitude from $10 \mathrm{MeV}$ to 20 $\mathrm{GeV}$ and demonstrates a very sharp "turn-over" at 700-800 MeV. The energy spectrum remains very hard up to $\sim 800 \mathrm{MeV}$ (with a power index $\sim-1$ ) and extended until tens of $\mathrm{GeV}$ with a power index between $\sim-5$ and -6 .

\section{CRD SPACE EDUCATION CENTER}

Artem Alikhanian, whose 100-th anniversary was celebrated at July 9, 2008 at the Nor-Amberd station, was not only brilliant scientist, but also an experienced educator. In early 60's, when the international contacts were still suppressed by soviet authorities, he initiated the famous Nor-Amberd schools, where problems of High-Energy and Elementary Particle Physics were discussed. Seasoned prominent and young new scientists from many countries participated in the activities of these schools. This tradition has been preserved up to the present days. CRD organizes in its Yerevan headquarters the Space Educational Center, where lectures on High-Energy Astrophysics, Cosmic Rays and Modeling of Physical processes are followed by experimental work in teaching laboratories, where students work with modern particle detectors and data acquisition electronics.

CRD developed an advanced Space Weather information product: Data Visualization Interactive Network (DVIN) for the Aragats Space Environmental Center. This product aims at visualizing scientific information about radiation conditions on the Earth caused by the strong radiation and geomagnetic storms from the sun. DVIN was officially announced as the world's best project in the e-science category at the World Summit on Information Society (WSIS) in Geneva in 2003. On June 10, 2005 DVIN was declared the winner of the Pan-Armenian e-content Mashtots-1600 competition.

CRD annually organizes international symposia devoted to Solar physics and Space Weather research. During the week of September 26-30, 2005, 75 scientists and students from 11 countries attended the second conference on Solar Extreme Events (SEE-2005) in Nor-Amberd, Armenia. Conference reports included information on consequences of Solar Extreme Events and Super Storms, the most violent explosions in the Solar System. The participants became acquainted with the ASEC monitors and capabilities of the Armenian physicists who created the Aragats Space Environmental Center. In September 28-October 3, 2008 the international symposium on Forecasting of the Radiation and Geomagnetic Storms by networks of particle detectors (FORGES-2008) took place in Nor-Amberd [40].

\section{FUTURE PLANS}

Currently Aragats is a modern scientific center, equipped with key scientific equipment and necessary supporting infrastructure, which is constantly being updated. Information on changing fluxes of secondary cosmic rays is distributed worldwide to numerous CRD collaborators. Modern science is impossible without a large-scale scientific cooperation. This cooperation is especially important for cosmic ray physics, which relies on data obtained with detectors located at different longitudes and latitudes all over the Earth, to develop a model of the solar-terrestrial connections. The Aragats and Nor-Amberd Neutron monitors are a part of the world-wide network of neutron monitors, solar neutron telescopes and muon detectors. 
Recently 12 countries of Europe decide to form joint data base for 1-minute counts from neutron monitors (NMBD), supported by European FP7 program. The joint project of muon detectors is currently implemented in collaboration with Germany, Switzerland and Israel. CRD initiated the development of a new world-wide particle detector network called "Space Environment Viewing and Analysis Network" (SEVAN) [41, 42]. The United Nations Office of Outer Space Affairs and the International Heliophysical Year (IHY) have launched a small instrument program as one of United Nations Basic Space Science (UNBSS) activity. SEVAN Network aims to improve the fundamental research on particle acceleration in vicinity of the Sun and space environment conditions. The new type of particle detectors will simultaneously measure changing fluxes of most species of secondary cosmic rays, thus turning into a powerful integrated device for exploration of solar modulation effects. The first SEVAN modules are under test at the Aragats Space Environmental Center in Armenia. The network will grow in 2009 with detectors deployed in Croatia and Bulgaria. In 2009 SEVAN detectors are planned to be installed in Slovakia, Germany and Costa Rica. Research groups from these countries participated in training on detector operation and data analysis during FORGES-2008 symposium.

The basic detecting unit of the SEVAN network (see Fig. 6) is assembled from standard slabs of $50 \times 50 \times 5 \mathrm{~cm}^{3}$ plastic scintillators. Between two identical assemblies of $100 \times 100 \times 5 \mathrm{~cm}^{3}$ scintillators (four standard slabs) are located two $100 \times 100 \times 5 \mathrm{~cm}^{3}$ lead absorbers and thick $50 \times 50 \times 25 \mathrm{~cm}^{3}$ scintillator assembly (5 standard slabs). A scintillator light capture cone and Photo Multiplier Tube (PMT) are located on the top, bottom and in the intermediate layer of the detector.

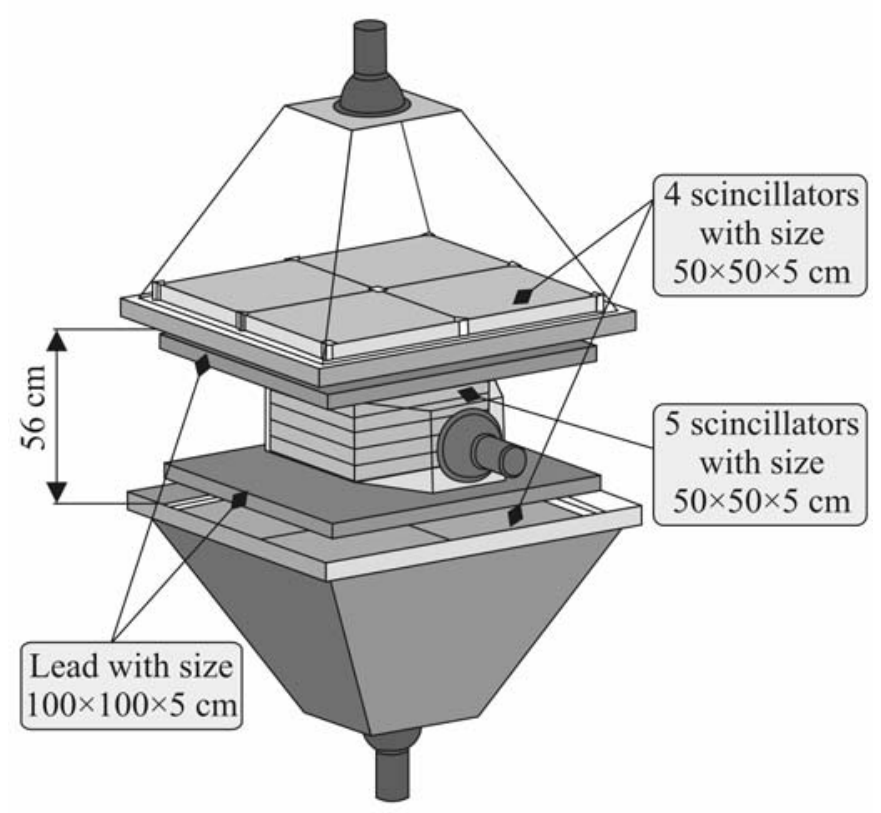

Fig. 6. Layout of the basic model of SEVAN network.

Incoming neutral particles undergo nuclear reactions in the thick $25 \mathrm{~cm}$ plastic scintillator and produce protons and other charged particles. In the upper 5-cm thick scintillator charged particles are registered very effectively; however, for the nuclear interactions of neutral particles there is not enough substance. When a neutral particle traverses the top thin $(5 \mathrm{~cm})$ scintillator, usually no signal is produced. The absence of the signal in the upper scintillators, coinciding with the signal in the middle scintillator, points to neutral particle detection. The coincidence of signals from the top and bottom scintillators indicates the traversal of high-energy muons. Microcontroller-based DAQ electronics and an Advanced Data Analysis System (ADAS) provide registration and storage of all logical combinations of the detector signals for further off-line analysis and for on-line alerts. The special ADAS sub-system allows the remote control of the PMT high voltage and of other important parameters of the DAQ electronics.

The network of hybrid particle detectors, measuring neutral and charged fluxes, provides the following advantages over existing detector networks measuring single species of secondary cosmic rays:

- Enlarged statistical accuracy of measurements;

- Probe different populations of primary cosmic rays with rigidities from $7 \mathrm{GV}$ up to $20 \mathrm{GV}$; 
- Reconstruct SCR spectra and determine position of the spectral "knees";

- Classify GLEs in "neutron" or "proton" initiated events;

- Estimate and analyze correlation matrices among different fluxes;

- Significantly enlarge the reliability of Space Weather alerts due to detection of 3 type particle fluxes instead of only one in existing neutron monitor and muon telescope world-wide networks.

A new trend in astrophysics research is in the observation of celestial objects in several wavelengths simultaneously (for example, in radio, optical, X-ray, and gamma-rays). A variety of complementary measurements give sufficient information for building and testing models of the galaxy formation, of supernovae explosions, of accompanying gamma-ray bursts, of accretion disc interactions with superdense objects, and finally of the evolution of the Universe itself. The additional information about the particles of highest energies arriving at the Solar system significantly enlarges the information on the most violent processes in the Universe.

Summarizing the situation with investigation of the CR spectra in the energy interval lasting from $10^{4}$ until $10^{20} \mathrm{eV}$ we can say:

- Lowest energies from $\mathrm{keV}$ to tens $\mathrm{TeV}$ region are rather well measured by space-born/air-born spectrometers located at satellites, space stations and balloons;

- The "knee" region spectra from $10^{14} \mathrm{eV}$ to $10^{17} \mathrm{eV}$ have been well explored during last 40 years by the surface arrays covering thousands of square meters;

- The ultra-high energy region - above $10^{19} \mathrm{eV}$ - after pioneering research of Haverah Park, VolcanoRanch, Yakutsk, AGASA and HIGHRES detectors took a mature state with AUGER-Sough observatory started to present valuable data, to be confirmed in next decade by an expected large volume of data.

This picture contains 2 obvious gaps in satisfactory well established spectra: $10^{13}-10^{14} \mathrm{eV}$ and $10^{17}-10^{19} \mathrm{eV}$. If the first gap can be filled with planned long-duration balloon flight and experiments on the Space Station, the second can be filled by the several square kilometer size particle arrays.

Recently CRD physicists started to prepare proposal of new large EAS surface array with the main scientific goal of measuring partial energy spectra of the cosmic rays in the poorly explored energy region of $10^{17}-10^{19} \mathrm{eV}$. The aim of the project is to build a large detector for investigation of the mentioned energy region, using already operating particle detectors on the slopes of Mt. Aragats and by installing new hybrid particle detectors measuring neutral and charged CR secondary fluxes. The main physical problem is determination of the contribution of the extragalactic CR component to give a consistent description for the entire GCR spectrum after the "knee".

The energy region of $10^{17}-10^{19} \mathrm{eV}$ is still poorly explored and the origin of the extragalactic cosmic rays is still mystery. To measure partial energy spectra (spectra of "light" and "heavy" nuclei groups) a very large area of EAS detection is required (at least several square kilometers). The optimal altitude (to measure maximal number of particles in EAS) is $\sim 2000 \mathrm{~m}$ above sea level. At these altitudes the EAS from primary proton with the energy $10^{18} \mathrm{eV}$ will produce $6 \times 10^{8}$ electrons. Therefore, also taking into account very severe climatic conditions at the altitude of $3200 \mathrm{~m}$ at the Aragats station, we propose to build a new large EAS detector in the Nor-Amberd-Byurakan region. We plan to use new-type of hybrid particle detectors measuring electron, muon and neutron contents of EAS at 2 sites separated by $\sim 3.5 \mathrm{~km}$ : at the Nor-Amberd research station and in the Antarut village.

Project objectives include:

- Development of a new-generation particle detector for measuring neutral and charged CR fluxes and their directions;

- Creation of a particle detector network for continuous detection of cosmic rays in the energy range $10^{17}-10^{19} \mathrm{eV}$;

- Determination of the characteristics of the "iron knee";

- Search for point sources of cosmic rays;

- Investigation of the "fine structure" of the partial energy spectra;

- Correlating obtained results with data from X-ray and $\gamma$-ray observatories, as well as from optical telescopes.

Two networks of particle detectors will be formed around the central part of $\sim 20 \mathrm{~m}^{2}$ hybrid particle detectors. Each array will be populated with detectors as soon as particle detectors are commissioned and assembled. The third site will be formed by the particle detectors of MAKET-ANI and GAMMA EAS arrays operating at the Aragats research station of Alikhanian Yerevan Physics Institute. All 3 sites have total area $\sim 0.35 \mathrm{~km}^{2}$ and will detect primary particles with energies up to several units of $10^{17} \mathrm{eV}$ (trigger 
conditions and corresponding EAS core collecting area will be obtained via Monte-Carlo simulations). Huge events triggering 2 arrays out of 3 will indicate primary energies above $10^{19} \mathrm{eV}$. EAS core collection area will be $\sim 15 \mathrm{~km}^{2}$ and $75 \mathrm{~km}^{2}$, correspondingly, for 2 and $5 \mathrm{~km}$ radii circles. Basic detectors tests and deployments will start in 2009; with appropriate funding in 2011 a new EAS detector will be equipped with enough modules for enlarging the investigated energy range up to $10^{18} \mathrm{eV}$.

Cosmic Ray research in the energy range of $10^{17}-10^{19} \mathrm{eV}$ is a continuation of the MAKET-ANI and GAMMA arrays energy domain of $10^{14}-10^{17} \mathrm{eV}$, thus providing continuous partial energy spectra in the energy range covering 5 orders of magnitude where almost all the significant features of energy spectra are located. No operating or planned surface array is intended to cover this very important and large energy domain: the KASCADE energy limit is $\sim 10^{18} \mathrm{eV}$, the energy ranges of HighRes and Auger start from $5 \times 10^{18} \mathrm{eV}$. Therefore the proposed detector will provide unique information extending the already well-investigated low-energy domain with the enigmatic highest energy domain.

\section{CONCLUSION}

The CRD staff includes approximately 80 people, who work at the Aragats and Nor-Amberd high altitude stations and at the headquarters in Yerevan where most of the data analysis and computation take place. Many of the staff members are young graduate students or recent postgraduates. Scientific research on Mt. Aragats is constantly searching for new methods and new frontiers as the Armenian physicists do their best in the quest of solving the mysteries of the Universe.

\section{ACKNOWLEDGMENTS}

We thank the former and the present CRD employees for their dedicated work at Aragats as well as our collaborators and supporters all over the world.

\section{REFERENCES}

1. Kocharyan, N.M., Yerevan State University, Scientific studies, 1940, vol. 12, p. 23.

2. Alikhanian, A.I., Alikhanov, A.I., and Nikitin, S.A., J. Phys., 1945, vol. 9, p. 175.

3. Alikhanian, A.I. and Alikhanov, A.I., J. Phys., 1945, vol. 9, p. 167.

4. Anderson, C.D. and Neddermeyer, S.H., Phys. Rev., 1937, vol. 51, p. 884.

5. Grigorov, N.L., Murzin, V.S., and Rapoport, I.D., ZhETF, 1958, vol. 7, p. 348.

6. Kocharian, N.M., Saakian, G.S., Kirakosyan, Z.A., et al., ZhETF, 1958, vol. 35, p. 1335.

7. Alikhanian, A.I. and Alikhanov, A.I., ZhETF, 1951, vol. 21, p. 1023.

8. Seife, C., Science, 2004, vol. 306, p. 1281.

9. Grigorov, N.L., Nesterov, V.E., and Rapoport, I.D., Yad. Phys., 1970, vol. 11, p. 1058.

10. Babayan, Kh.P., Grigorov, N.L., Mamidjanian, E.A., et al., Izv. AN SSSR, ser. fiz., 1965, vol 30, p. 1652.

11. Azaryan, M.O., Zazyan, M.Z., and Mamidjanian, E.A., Yad. Phys., 1977, vol. 26, p. 141.

12. Avakian, V.V., Mamidjanian, E.A., and Oganesyan, A.G., Izv. AN SSSR, ser. fiz., 1978, vol. 40, p. 1058.

13. Asatiani, T.L., Chilingarian, A.A., et al., Izv. AN SSSR, ser. fiz., 1980, vol. 45, p. 323.

14. Alikhanian A.I., Avakian V.V., Mamidjanian E.A., et al., Izv. AN SSSR, ser.fiz., 1974, vol. 38, p. 1993.

15. Danilova, T.V., Dunaevsky, A.M., Erlykin, A.D., et al., Izv. AN SSSR, ser. fiz., 1982, vol. 17, p. 129.

16. Avakian, V.V., Bazarov, E.V., et al., VANT, ser. Tech. Phys. Exp., 1986, vol. 5, p. 1.

17. Garyaka, A.P., Martirosov, R.M., et al., J. Phys. G: Nucl. Part., 2002, vol. 28, p. 231.

18. Chilingarian, A.A., Computer Physics Communications, 1989, vol. 54, p. 381.

19. Chilingaryan, A., Neurocomputing, 1994, vol. 6, p. 497.

20. Antoni, T., Apel, W.D., Badea F., et al., Nucl. Instr. Meth. A, 2003, vol. 513, p. 490.

21. Chilingarian, A., Gharagyozyan, G., Hovsepyan, G., et al., Astroph. Journal, 2004, vol. 603, p. L23.

22. Chilingarian, A.A., Hovsepyan, G.G., Melkymyan, L.G., et al., Astropart. Phys., 2007, vol. 28 , p. 58.

23. Antoni, T., Apel, W.D., Badea, F., et al., Astropart. Phys., 2005, vol. 24, p. 1.

24. Horns, D. and Rohring, A., Proc. 27th ICRC, Hamburg, Germany, 2001, vol. 1, p. 1091.

25. Aglietta, M., Alessandro, B., Antonioli, P., et al., Astropart. Phys., 2004, vol. 20, p. 641.

26. Uchiyama, Y., Aharonyan, F., Tanaka, T., et al., Nature Letters, 2007, vol. 449/4, 10.1038/nature 06210.

27. Takahashi, T., Tanaka, T., Uchiyama, Y., et al., arXiv:0708.2002v1 (astro-ph), 2002.

28. Aharonyan, F.A., Akhperjanian, A.G., Bazer-Bachi, A.R., et al. Astron. Astrophys., 2007, vol. 464, p. 235. 
29. Mirzoyan, R. et al., Nucl. Instr. Meth. A, 1994, vol. 351, p. 513.

30. Chilingarian, A., Melkumyan, L., Hovsepyan, G., and Reymers, A., Nucl. Instr. Meth. A, 2007, vol. 574, p. 255.

31. Chilingarian, A.A., Avagyan, K., Babayan, V., et al., J. Phys. G: Nucl. Part. Phys., 2003, vol. 29, p. 939.

32. Chilingarian, A.A., Arakelyan, K., Avagyan, K., et al., Nucl. Instr. Meth. A, 2005, vol. 543, p. 483.

33. Bostanjyan, N.K., Chilingarian, A.A., et al., Advances in Space Research, 2007, vol. 39, p. 1456.

34. Chilingarian, A.A. and Reymers, A.E., Astropart. Phys., 2007, vol. 27, p. 465.

35. Chilingarian, A.A., Advances of Space Research, doi: 10.1016/j.asr.2008.10.005.

36. Miyasaka, H., Takahashi, E., et al., Proc. 29th ICRC, Pune, India, 2005, vol. 1, p. 241.

37. Zhu, F.R., Tang, Y.Q., Zhang, Y., et al., Proc. 29th ICRC, Pune, India, 2005, vol. 1, p. 185.

38. Karpov, S.N., Karpova, Z.M., Balabin, Yu.V., and Vashenyuk, E.V., Proc. 29th ICRC, Pune, India, 2005, vol. 1, p. 193.

39. D'Andrea, C. and Poirier, J., Geophys. Res. Lett., 2005, vol. 32, p. L14102, doi:10.1029/2005GL023336.

40. Chilingarian, A.A., Workshop, Space Research Today, 2008, vol. 173, p. 125.

41. Chilingarian, A.A. and Reymers, A., Ann. Geophys., 2008, vol. 26, p. 249.

42. Chilingarian, A.A., Avakyan, K., Arakelyan, K., et al., Earth, Moon, and Planets, DOI: 10.1007/s11038-0089288-1, 2009. 